\title{
THE FIXED POINT THEOREM IN EQUIVARIANT COHOMOLOGY
}

\author{
J. D. S. JONES AND S. B. PETRACK
}

\begin{abstract}
In this paper we study the $S^{1}$-equivariant de Rham cohomology of infinite dimensional $S^{1}$-manifolds. Our main example is the free loop space $L X$ where $X$ is a finite dimensional manifold with the circle acting by rotating loops. We construct a new form of equivariant cohomology $h_{T}^{*}$ which agrees with the usual periodic equivariant cohomology in finite dimensions and we prove a suitable analogue of the classical fixed point theorem which is valid for loop spaces $L X$. This gives a cohomological framework for studying differential forms on loop spaces and we apply these methods to various questions which arise from the work of Witten [16], Atiyah [2], and Bismut [5]. In particular we show, following Atiyah in [2], that the $\hat{A}$-polynomial of $X$ arises as an equivariant characteristic class, in the theory $h_{T}^{*}$, of the normal bundle to $X$, considered as the space of constant loops, in $L X$.
\end{abstract}

\section{INTRODUCTION}

The aim of this paper is to provide a cohomological framework for studying the questions concerning differential forms on loop spaces which arise from the work of Witten [16], Atiyah [2] and Bismut [5]. We prove a suitable analogue of the classical fixed point theorem of $S^{1}$-equivariant cohomology which is valid in the case of free loop spaces $L X$ with the circle acting by rotating loops. In addition, we construct an equivariant Thom/Euler class (see $\S 4$ ) for the normal bundle of the submanifold of constant loops $X$ in the space of all loops $L X$ and show how this class is related to the $\hat{A}$ polynomial of $X$. These results were announced, without proof, in [12].

Throughout we will work with complex valued differential forms and de Rham cohomology. This is dictated by simplicity and our applications. Let $Y$ be a smooth manifold equipped with a smooth $S^{1}$ action. We will refer to $Y$ as a smooth $S^{1}$-manifold. We can associate to $Y$ its equivariant cohomology $H_{T}^{*}(Y)$. This is a module over the coefficient ring $H_{T}^{*}$ (point $)=\mathbb{C}[u]$ where $u$ is an indeterminate of degree 2 . Equivariant cohomology can be localised by inverting the multiplicative set $\left\{1, u, u^{2}, \ldots\right\}$ to form periodic equivariant cohomology $u^{-1} H_{T}^{*}(Y)$. The classical fixed point theorem says that if $Y$ is finite dimensional and $F$ is the fixed point set of the $S^{1}$ action on $Y$ then the

Received by the editors April 15, 1989.

1980 Mathematics Subject Classification (1985 Revision). Primary 58A10, 53C80, 55N35, $55 \mathrm{~N} 91,58 \mathrm{~B} 05$. 
inclusion $i: F \rightarrow Y$ induces an isomorphism

$$
i^{*}: u^{-1} H_{T}^{*}(Y) \rightarrow u^{-1} H_{T}^{*}(F)
$$

It is straightforward to see that $u^{-1} H_{T}^{*}(F)=H^{*}(F) \otimes \mathbb{C}\left[u, u^{-1}\right]$ where $H^{*}(F)$ is the ordinary de Rham cohomology of $F$ and so we get a computation of periodic equivariant cohomology in terms of the cohomology of the fixed point set.

Now let $X$ be a smooth, connected, finite dimensional manifold and let $L X$ be the space of all smooth loops in $X$ with its $C^{\infty}$ topology. The space $L X$ is an infinite dimensional manifold modelled on a Fréchet space. The circle acts smoothly on $L X$ and the fixed point set of this action is precisley the manifold $X$, considered as the space of constant loops. According to Goodwillie [10], $u^{-1} H_{T}^{*}(L X)$ depends only on $\pi_{1}(X)$ so that the fixed point theorem, as it stands, cannot be true for $L X$. This has been an obstacle to progress in the study of differential forms and integration on $L X$.

Our solution to this difficulty is to construct a new form of equivariant cohomology, denoted $h_{T}^{*}(Y)$, which will be used in place of $u^{-1} H_{T}^{*}(Y)$. The groups $h_{T}^{*}(Y)$ are defined by a simple and natural modification of one definition of periodic equivariant cohomology. If $Y$ is finite dimensional then $h_{T}^{*}(Y)=u^{-1} H_{T}^{*}(Y)$ but in a large class of infinite dimensional examples, including the case $Y=L X$, the inclusion $i: F \rightarrow Y$ of the fixed point set induces an isomorphism $h_{T}^{*}(Y) \longrightarrow h_{T}^{*}(F)$. This cohomology theory is constructed in $\S 1$ and the fixed point theorem is stated precisely in $\S 2$. Some alternative theories which also satisfy the fixed point theorem are discussed in $\S 3$. In $\S 4$ we show how to construct an inverse, at the level of differential forms, for the restriction homomorphism $i^{*}$ and following [3, 6, 4, and 7] explain how this leads to integration formulas. The most important ingredient is the construction of an equivariant form $\tau$, essentially the form $\exp (-(\omega+E))$ of $[2,6]$, which defines a class in $h_{T}^{*}(Y)$ and has the key property that $i^{*}(\tau)=1$. This form $\tau$ is essentially the equivariant Thom/Euler class of the normal bundle to the fixed point set. In $\S 5$ we show how the form $\tau$ on $L X$, is related to the $\hat{A}$ polynomial of $X$, compare [2].

The main theme running through this article is that if we use the cohomology theory $h_{T}^{*}$ many of the familiar results concerning the equivariant cohomology of finite dimensional manifolds generalise, in a fairly straightforward manner, to infinite dimensions. The fixed point theorem (Theorem 2.1) and the way in which the Thom/Euler class $\tau$ of the normal bundle to the space of constant loops gives rise to the $\hat{A}$ polynomial (see $\S 5$ ) are the main justifications for our equivariant cohomology theory $h_{T}^{*}$. Our ultimate goal is to use $h_{T}^{*}$ to relate the differential geometry of loop spaces to index theory of elliptic differential operators. This is a more delicate question requiring the use of ideas from cyclic homology which will be treated elsewhere, see [8, 9]. 
Remark on conventions. As we are interested primarily in infinite dimensional manifolds, we should state at the outset the structures we assume implicit in the word manifold. All manifolds will be assumed to admit smooth partitions of unity and a smoothly varying positive definite inner product (that is a preHilbert space structure) on each tangent space. We could in fact prove our theorems under weaker conditions but these hypotheses simplify the presentation and are verified in the geometric examples.

\section{EQUIVARIANT COHOMOLOGY THEORIES}

Let $Y$ be a smooth $S^{1}$-manifold and let $\Omega^{*}(Y)$ be the space of smooth differential forms on $Y$. Let $l: \Omega^{n}(Y) \rightarrow \Omega^{n-1}(Y)$ be the operator given by taking the interior product with the vector field on $Y$ generating the circle action. It is easy to check that $l^{2}=0$. Let $\Omega_{T}^{*}(Y)$ be the space of invariant forms on $Y$. Let $d$ be the exterior derivative, then $d l+l d=0$ on $\Omega_{T}^{*}(Y)$.

The following de Rham model for equivariant cohomology is described in [3]. Form the polynomial ring $\Omega_{T}^{*}(Y)[u]$ where $u$ is an indeterminate of degree 2 . Introduce the operator $d_{T}=d+u l$ on $\Omega_{T}^{*}(Y)[u]$; then $d_{T}^{2}=0$ and $d_{T}$ is a derivation with respect to the natural product on $\Omega_{T}^{*}(Y)[u]$. Equivariant cohomology is defined by

$$
H_{T}^{*}(Y)=H^{*}\left(\Omega_{T}^{*}(Y)[u] ; d_{T}\right) .
$$

If we use $\Omega_{T}^{*}(Y)\left[u, u^{-1}\right]$, the space of Laurent polynomials in $u, u^{-1}$, in place of $\Omega_{T}^{*}(Y)[u]$ then we get periodic equivariant cohomology:

$$
u^{-1} H_{T}^{*}(Y)=H^{*}\left(\Omega_{T}^{*}(Y)\left[u, u^{-1}\right] ; d_{T}\right) .
$$

This is easy to check using the fact that localisation and forming homology commute.

The cohomology theory $h_{T}^{*}$ is defined using the space $\Omega_{T}^{*}(Y)\left[\left[u, u^{-1}\right]\right]$ of formal power series in $u, u^{-1}$ with coefficients in $\Omega_{T}^{*}(Y)$. A typical homogeneous element $\omega$, of total degree $p$, in $\Omega_{T}^{*}(Y)\left[\left[u, u^{-1}\right]\right]$ has the form

$$
\omega=\sum_{-\infty<i \leq p / 2} a_{i} u^{i}, \quad a_{i} \in \Omega_{T}^{p-2 i}(Y) .
$$

We can multiply homogeneous elements, so $\Omega_{T}^{*}(Y)\left[\left[u, u^{-1}\right]\right]$ becomes a graded ring and $d_{T}$ is a derivation with respect to this product. We define $h_{T}^{*}(Y)$ to be

$$
h_{T}^{*}(Y)=H^{*}\left(\Omega_{T}^{*}(Y)\left[\left[u, u^{-1}\right]\right] ; d_{T}\right)
$$

and we will refer to this theory as completed periodic equivariant cohomology. The presence of an infinite number of negative powers of $u$ is essential; if we insist on only a finite number of negative powers then we get periodic equivariant cohomology and we know from [10] that this is trivial in the infinite dimensional cases of interest. If $Y$ is finite dimensional then $\Omega_{T}^{*}(Y)[u]=\Omega_{T}^{*}(Y)\left[\left[u, u^{-1}\right]\right]$ 
and so $h_{T}^{*}(Y)=u^{-1} H_{T}^{*}(Y)$ but if $Y$ is infinite dimensional the two theories are, in general, different.

We will often use the term equivariant differential form for an element of one of the algebras $\Omega_{T}^{*}(Y)[u], \Omega_{T}^{*}(Y)\left[u, u^{-1}\right], \Omega_{T}^{*}(Y)\left[\left[u, u^{-1}\right]\right]$ and refer to the operator $d_{T}$ as the equivariant exterior derivative. Then terms like equivariantly closed will have the natural meaning.

The cohomological properties of $h_{T}^{*}$ differ from those of the classical theories in one important way. The theories $H_{T}^{*}$ and $u^{-1} H_{T}^{*}$ have the following weak homotopy invariance property: Suppose that $f: X \rightarrow Y$ is a smooth equivariant map which is an ordinary homotopy equivalence; then $f^{*}$ is an isomorphism in $H_{T}^{*}$ and $u^{-1} H_{T}^{*}$. The proof of this fact is a standard double complex argument, see for example [11]. The theory $h_{T}^{*}$ does not have this property as we will show by an example in $\S 2$. It does have the following equivariant homotopy invariance property. Suppose that $f_{0}, f_{1}: X \rightarrow Y$ are smooth equivariant maps which are equivariantly homotopic (i.e. there is a smooth equivariant map $F:[0,1] \times X \rightarrow Y$, where the circle acts trivially on $[0,1]$, such that $F(0, x)=f_{0}(x)$ and $\left.F(1, x)=f_{1}(x)\right)$; then $f_{0}^{*}=f_{1}^{*}$ in $h_{T}^{*}$. Of course the theories $H_{T}^{*}$ and $u^{-1} H_{T}^{*}$ also have this property.

To prove the equivariant homotopy invariance property define $s=\pi_{*} F^{*}$ where $\pi_{*}$ is integration along the fibres of the projection $\pi:[0,1] \times X \rightarrow X$. Then $d s+s d=f_{0}^{*}-f_{1}^{*}$ and the fact that $F$ is equivariant shows that $s$ maps invariant forms to invariant forms and $s l+l s=0$. Therefore

$$
d_{T} s+s d_{T}=f_{0}^{*}-f_{1}^{*}
$$

and so $f_{0}^{*}=f_{1}^{*}$ in each of the three equivariant cohomology theories.

There are Mayer-Vietoris sequences, associated to two invariant open sets $U$ and $V$ such that $U \cup V=Y$, in each of the equivariant cohomology theories. Since the circle is compact we may average a chosen partition of unity to construct invariant partitions of unity and then use the standard construction of the Mayer-Vietoris sequence.

Here is a slightly different description of the groups $h_{T}^{*}$. Let

$$
\Omega_{T}(Y)=\prod_{q \geq 0} \Omega_{T}^{q}(Y), \quad \Omega_{T}^{\mathrm{ev}}(Y)=\prod_{q \geq 0} \Omega_{T}^{2 q}(Y), \quad \Omega_{T}^{\mathrm{odd}}(Y)=\prod_{q \geq 0} \Omega_{T}^{2 q+1}(Y)
$$

and set $\delta: \Omega_{T}(Y) \rightarrow \Omega_{T}(Y)$ to be the operator $\delta=d+l$. This amounts to setting $u=1$. Then $\delta^{2}=0$ and we have

$$
\begin{aligned}
& h_{T}^{2 n}(Y)=\frac{\operatorname{ker}\left(\delta: \Omega_{T}^{\mathrm{ev}}(Y) \longrightarrow \Omega_{T}^{\mathrm{odd}}(Y)\right)}{\operatorname{im}\left(\delta: \Omega_{T}^{\mathrm{odd}}(Y) \longrightarrow \Omega_{T}^{\mathrm{ev}}(Y)\right)}, \\
& h_{T}^{2 n+1}(Y)=\frac{\operatorname{ker}\left(\delta: \Omega_{T}^{\mathrm{odd}}(Y) \longrightarrow \Omega_{T}^{\mathrm{ev}}(Y)\right)}{\operatorname{im}\left(\delta: \Omega_{T}^{\mathrm{ev}}(Y) \longrightarrow \Omega_{T}^{\mathrm{odd}}(Y)\right)} .
\end{aligned}
$$




\section{THE FIXED POINT THEOREM}

Let $Y$ be a smooth $S^{1}$-manifold; then the action will be called regular if the fixed point set $F$ is a smooth submanifold which has an invariant neighbourhood $N$ such that the inclusion $i: F \rightarrow N$ is an equivariant homotopy equivalence. If $Y$ is finite dimensional then any smooth action of the circle is regular. If the fixed point set has an invariant tubular neighbourhood, by which we mean there is an $S^{1}$-vector bundle $E$ over $F$ and a smooth equivariant map $h: E \rightarrow Y$ which is a diffeomorphism onto its image, then the action is regular. We will construct such a neighbourhood for $L X$ in an Appendix. The question of finding general conditions under which an action of the circle on an infinite dimensional manifold is regular is technical and will not be pursued here.

Theorem 2.1. If $Y$ is a regular $S^{1}$-manifold then the inclusion of the fixed point set $i: F \rightarrow Y$ induces an isomorphism

$$
i^{*}: h_{T}^{*}(Y) \rightarrow h_{T}^{*}(F)
$$

Using the notation introduced in $\S 1, h_{T}^{*}(F)=H^{*}(F)\left[\left[u^{-1}, u\right]\right]$ and so we get a computation of $h_{T}^{*}(Y)$ in terms of $H^{*}(F)$ just as in the case of the classical fixed point theorem. The most important step in the proof of Theorem 1.2 is the following lemma.

Lemma 2.2. Let $Y$ be a smooth $S^{1}$-manifold and suppose that the circle action on $Y$ has no fixed points; then $h_{T}^{*}(Y)=0$.

Proof. We construct an equivariant form $\omega \in \Omega_{T}^{*}(Y)\left[\left[u, u^{-1}\right]\right]$ such that $d_{T} \omega$ $=1$. Using the fact that $d_{T}$ is a derivation with respect to the natural product on $\Omega_{T}^{*}(Y)\left[\left[u, u^{-1}\right]\right]$ it follows that $s(x)=\omega x$ is a contracting homotopy of the complex $\left(\Omega_{T}^{*}(Y)\left[\left[u, u^{-1}\right]\right] ; d_{T}\right)$; that is

$$
d_{T} s(x)+s d_{T}(x)=x \text {. }
$$

This shows that $h_{T}^{*}(Y)=0$. It remains to construct the form $\omega$.

Let $V$ be the vector field on $Y$ generating the circle action and let $\alpha$ be the one-form dual to $V$; then

$$
d_{T} \alpha=d \alpha+u l \alpha=d \alpha+u|V|^{2}
$$

Since the circle action has no fixed points, $V$ is never zero and

$$
d_{T^{\alpha}}=u|V|^{2}\left(1+\frac{d \alpha}{u|V|^{2}}\right) \text {. }
$$

Therefore $d_{T} \alpha$ is a unit in the ring $\Omega_{T}^{*}(Y)\left[\left[u, u^{-1}\right]\right]$; the inverse is given by

$$
\gamma=\left(d_{T} \alpha\right)^{-1}=u^{-1}|V|^{-2} \sum_{i=0}^{\infty}\left(\frac{-d \alpha}{u|V|^{2}}\right)^{i} .
$$


Since $\gamma\left(d_{T} \alpha\right)=1$ it follows that $\left(d_{T} \gamma\right)\left(d_{T} \alpha\right)=0$ and therefore, since $d_{T} \alpha$ is a unit, $d_{T} \gamma=0$; so $d_{T}(\alpha \gamma)=\left(d_{T} \alpha\right) \gamma=1$ and we define $\omega=\alpha \gamma$. $u^{-1}$.

Note that in finite dimensions $d \alpha$ is nilpotent so that $\gamma$ is a polynomial in

Proof of Theorem 2.1. Pick an invariant neighbourhood $N$ of $F$ such that the inclusion of $F$ in $N$ is an equivariant homotopy equivalence. We use the Mayer-Vietoris sequence associated to the invariant open sets $Y \backslash F$ and $N$. Both $Y \backslash F$ and $(Y \backslash F) \cap N$ are fixed point free so, using Lemma 2.2 , the sequence gives an isomorphism

$$
i_{N}^{*}: h_{T}^{*}(Y) \rightarrow h_{T}^{*}(N)
$$

The inclusion of $F$ in $N$ is an equivariant homotopy equivalence and the equivariant homotopy invariance property completes the proof.

It is important to note that the fixed point theorem and the weak homotopy invariance property are incompatible in infinite dimensions. There is no equivariant cohomology theory $k^{*}$ with the following properties:

(1) The weak homotopy invariance property described in $\S 1$.

(2) $k^{*}$ (point) $\neq 0$.

(3) $k^{*}(Y)=0$ if $Y$ is fixed point free.

To see this let $S$ be the unit sphere in a separable Hilbert space with the circle action given by multiplication by unit complex numbers. This is a standard model for $E S^{1}$. The constant map $S \rightarrow$ point is equivariant and since $S$ is contractible it is an ordinary homotopy equivalence. Therefore (1) and (2) show that $k^{*}(S) \neq 0$. However $S$ is fixed point free and so this contradicts (3).

Therefore in infinite dimensions we must choose between the fixed point theorem and the weak homotopy invariance property.

\section{OTHER DE RHAM MODELS FOR EQUIVARIANT COHOMOLOGY}

There are other de Rham models for equivariant cohomology which use differential forms in an essential way. Here we describe and compare these variations. First we can replace the space of invariant forms on the $S^{1}$-manifold $Y$ by the space of all forms $\Omega^{*}(Y)$ and then replace the operator $l$ by the operator $I: \Omega^{p}(Y) \longrightarrow \Omega^{p-1}(Y)$ defined by the following procedure. The circle action defines a smooth map $\varphi: S^{1} \times Y \rightarrow Y$ and $I$ is defined to be

$$
I=\pi_{*} \varphi^{*}: \Omega^{p}(Y) \rightarrow \Omega^{p-1}(Y)
$$

where $\pi_{*}$ is integration along the fibres of the projection $\pi: S^{1} \times Y \rightarrow Y$. It is straightforward to check that $I=l A=A l$ where $A: \Omega^{*}(Y) \rightarrow \Omega_{T}^{*}(Y)$ is the 
averaging operator. Furthermore $I$ satisfies the identities $I^{2}=d I+I d=0$ and so we may form the complexes

$\left(\Omega^{*}(Y)[u] ; d+u I\right), \quad\left(\Omega^{*}(Y)\left[u, u^{-1}\right] ; d+u I\right), \quad\left(\Omega^{*}(Y)\left[\left[u, u^{-1}\right]\right] ; d+u I\right)$.

From the definition of $I$ it follows that if $\alpha \in \Omega_{T}^{*}(Y)$ then $I \alpha=\imath \alpha$.

Theorem 3.1. The inclusion $j: \Omega_{T}^{*}(Y) \rightarrow \Omega^{*}(Y)$ induces isomorphisms

$$
\begin{aligned}
& H^{*}\left(\Omega_{T}^{*}(Y)[u] ; d_{T}\right) \rightarrow H^{*}\left(\Omega^{*}(Y)[u] ; d+u I\right), \\
& H^{*}\left(\Omega_{T}^{*}(Y)\left[u, u^{-1}\right] ; d_{T}\right) \rightarrow H^{*}\left(\Omega^{*}(Y)\left[u, u^{-1}\right] ; d+u I\right), \\
& H^{*}\left(\Omega_{T}^{*}(Y)\left[\left[u, u^{-1}\right]\right] ; d_{T}\right) \rightarrow H^{*}\left(\Omega^{*}(Y)\left[\left[u, u^{-1}\right]\right] ; d+u I\right) .
\end{aligned}
$$

Proof. Since $S^{1}$ is compact and connected the de Rham cohomology of $Y$ can be computed from the complex of invariant forms. So the first and second isomorphisms, and the third in the case where $Y$ is finite dimensional, follow from standard double complex arguments, see for example [11]. But we need an explicit homotopy argument for the third isomorphism in the case where $Y$ is infinite dimensional. This is an allusion to the more analytical nature of the theory $h_{T}^{*}$.

Since $A I=l A$, the averaging operator $A$ defines a map from the complexes using all forms to the complexes using invariant forms. The composite $A j$ is the identity so to complete the proof we construct an operator $s: \Omega^{*}(Y) \rightarrow \Omega^{*}(Y)$ with the following properties:

(1) $d s-s d=1-j A$,

(2) $s I=I s$.

It will then follow that $(d+u I) s-s(d+u I)=1-j A$. Therefore $j A$ is homotopic to the identity and $j, A$ induce inverse isomorphisms in cohomology.

To define $s$ regard the circle action as a one-parameter group of diffeomorphisms $\varphi_{t}$ of $Y$ with $\varphi_{0}=\varphi_{1}$. Define $h_{t}:[0, t] \times Y \rightarrow Y$ by setting $h_{t}(u, y)=\varphi_{u}(y)$. If $\omega \in \Omega^{*}(Y)$ define $s(\omega)$ as follows:

$$
s(\omega)=\int_{0}^{1}\left(\pi_{t}\right)_{*} h_{t}^{*}(\omega) d t
$$

where $\left(\pi_{t}\right)_{*}$ is integration along the fibres of the projection $\pi_{t}:[0, t] \times Y \rightarrow Y$. To compute $d s(\omega)$ use the formula

$$
d\left(\left(\pi_{t}\right)_{*} \theta\right)=\left(\pi_{t}\right)_{*}(d \theta)+\left(i_{0}^{*} \theta-i_{t}^{*} \theta\right)
$$

where $i_{0}, i_{t}: Y \rightarrow[0, t] \times Y$ are the inclusions of the two ends. This gives

$$
\begin{aligned}
d s(\omega) & =\int_{0}^{1} d\left(\left(\pi_{t}\right)_{*} h_{t}^{*}(\omega)\right) d t \\
& =\int_{0}^{1}\left(\pi_{t}\right)_{*} h_{t}^{*}(d \omega) d t+\int_{0}^{1}\left(\varphi_{0}^{*} \omega-\varphi_{t}^{*} \omega\right) d t \\
& =s(d \omega)+\omega-j A(\omega) .
\end{aligned}
$$

It is straightforward to check that $I s(\omega)=s I(\omega)$. 
The second variation is to use finitely smooth forms which are defined as follows. Let $\omega$ be a multi-linear function from the space of vector fields on $Y$ to functions on $Y$ which is multi-linear over functions. Then $\omega$ will be called finitely smooth if for any finite dimensional manifold $M$ and any smooth map $f: M \rightarrow Y$ the form $f^{*}(\omega)$ is a smooth form on $M$. This is only interesting in infinite dimensions. We can form the algebra $\Omega^{*}(Y)_{f}$ of finitely smooth forms. The operators $d, l$ and $I$ all map finitely smooth forms to finitely smooth forms and so we may form cohomology theories

$$
H_{T}^{*}(Y)_{f}, \quad u^{-1} H_{T}^{*}(Y)_{f}, \quad h_{T}^{*}(Y)_{f} .
$$

Since any smooth differential form is finitely smooth there are homomorphisms

$$
H_{T}^{*}(Y) \rightarrow H_{T}^{*}(Y)_{f}, \quad u^{-1} H_{T}^{*}(Y) \rightarrow u^{-1} H_{T}^{*}(Y)_{f}, \quad h_{T}^{*}(Y) \rightarrow h_{T}^{*}(Y)_{f} .
$$

It is not clear whether, in general, these maps are isomorphisms. However it is clear that our proof of the fixed point theorem, Theorem 2.1, extends to the theory $h_{T}^{*}(Y)_{f}$.

More generally, suppose $Y$ is equipped with an increasing filtration by invariant finite dimensional submanifolds $Y_{n}$. We define the space of filtered forms to be

$$
\text { Inv } \lim \Omega^{*}\left(Y_{n}\right)
$$

and then construct equivariant cohomology theories using these filtered forms. One important example, which occurs in Taubes' work on elliptic genera [15], where there is a natural filtration is the case where $Y$ is the normal bundle to the space of constant loops in the space of all loops. Here the filtration comes from the Fourier decomposition, see [2].

We will not pursue the theory of finitely smooth forms or filtered forms here since all the constructions we wish to do can be carried out directly in the space of smooth forms.

\section{THE INVERSE OF RESTRICTION TO THE FIXED POINT SET AND THE INTEGRATION FORMULA}

Let $Y$ be a $S^{1}$-manifold whose fixed point set $F$ has an invariant tubular neighbourhood. We will construct a map $\Omega_{T}^{*}(Y)\left[\left[u, u^{-1}\right]\right] \rightarrow \Omega_{T}^{*}(Y)\left[\left[u, u^{-1}\right]\right]$ which commutes with the equivariant exterior derivative such that the induced map $h_{T}^{*}(F) \rightarrow h_{T}^{*}(Y)$ is the inverse of $i^{*}$. We will also explain the relation between this construction and the integration formulas of $[3,6,4,7]$.

First consider the following general situation. Let $Y$ be an $S^{1}$-manifold with an invariant metric. We have the following geometric data:

(1) The smooth vector field $V$ generating the circle action.

(2) The one-form $\alpha$ dual to $V$ in the given metric.

(3) The two-form $\omega=d \alpha$.

(4) The function $H=l \alpha=|V|^{2}$.

(5) $\tau=\exp \left(-\left(H+u^{-1} \omega\right)\right) \in \Omega_{T}^{*}(Y)\left[\left[u, u^{-1}\right]\right]$. 
All this data is natural with respect to equivariant maps which preserve the metric. The equivariant form $\tau$ is homogeneous of total degree 0 , equivariantly closed and $i^{*}(\tau)=1$.

First we look at the case where $Y$ is a smooth $S^{1}$-vector bundle $E$ with an invariant metric over some manifold $X$ where the circle action on $X$ is trivial. Let $i: X \rightarrow E$ be the inclusion of the zero section and let $\pi: E \rightarrow X$ be the projection. Define

$$
\sigma: \Omega_{T}^{*}(X)\left[\left[u, u^{-1}\right]\right] \rightarrow \Omega_{T}^{*}(E)\left[\left[u, u^{-1}\right]\right], \quad \sigma(\alpha)=\tau \pi^{*}(\alpha) ;
$$

then $i^{*} \sigma=1$ and since $\tau$ is equivariantly closed, $\sigma$ commutes with $d_{T}$.

Now suppose that $Y$ is a $S^{1}$-manifold whose fixed point set has an invariant tubular neighbourhood. Let $E$ be the normal bundle of the fixed point set and let $h: E \rightarrow Y$ be an equivariant diffeomorphism of $E$ with a neighbourhood of $F$. We assume that $Y$ and $E$ have compatible invariant metrics. We can "graft" the equivariant forms $\tau$ and $\tau \pi^{*}(\alpha)$ in $\Omega_{T}^{*}(E)\left[\left[u, u^{-1}\right]\right]$ into $Y$ by the following proceedure: As in [14], let $g: E \rightarrow E$ be the map

$$
g(y)=\frac{y}{\sqrt{1+|y|^{2}}} .
$$

This is a fibrewise diffeomorphism of $E$ with the unit disc bundle in $E$. Using $g$ and extending by zero defines a smooth equivariant form $\tau_{1}$ on $E$ whose support is contained in the unit disc bundle. Now use $h$ and extend $\tau_{1}$ by zero to construct a smooth equivariant form $\tau_{h}$ on $Y$. By the same procedure we can define a smooth equivariant form $\sigma_{h}(\alpha)$ on $Y$.

It is necessary to do this grafting proceedure in two steps since we do not have enough hypotheses on the diffeomorphism $h: E \rightarrow h(E)$ to guarantee that the form constructed by using $h$ and extending by zero is smooth. Note that $h$ may be chosen so that $\tau_{h}$ and $\sigma_{h}(\alpha)$ have support in an arbitrarily small neighbourhood of the fixed point set.

Theorem 4.1. Let $Y$ be a $S^{1}$-manifold with fixed point set $F$ and suppose that $F$ has an invariant tubular neighbourhood. The map

$$
\sigma_{h}: \Omega^{*}(F)\left[\left[u, u^{-1}\right]\right] \rightarrow \Omega_{T}^{*}(Y)\left[\left[u, u^{-1}\right]\right]
$$

commutes with the equivariant exterior derivative and in cohomology

$$
\sigma_{h}=\left(i^{*}\right)^{-1}: h_{T}^{*}(F) \rightarrow h_{T}^{*}(Y) .
$$

Proof. The fact that $\sigma_{h}$ commutes with $d_{T}$ follows directly from the definitions. To prove the second statement we compute $i^{*} \sigma_{h}$ :

$$
i^{*} \sigma_{h}(\alpha)=i^{*}\left(\tau_{h} \pi^{*}(\alpha)\right)=i^{*}\left(\tau_{h}\right) \alpha=\alpha
$$

where the last equality follows since $i^{*}\left(\tau_{h}\right)=1$. 
Now we explain the relation between this theorem and integration formulas for equivariant differential forms in finite dimensions. First we will discuss the case of a finite dimensional $S^{1}$ vector bundle $\pi: E \rightarrow X$ over a compact manifold $X$ where the circle acts trivially on $X$. We assume that $X$ is equipped with a Riemannian metric and $E$ an invariant metric. This provides the manifold $E$ with an invariant Riemannian metric and so we have the equivariant form $\tau$ defined on $E$. Given any equivariant form $\alpha$ on $X$, $\tau \pi^{*}(\alpha)$ has exponential decay in the fibre directions and since $X$ is compact it follows $\tau \pi^{*}(\alpha)$ has a well defined integral. Furthermore we can define the integral along the fibres $\pi_{*}\left(\tau \pi^{*}(\alpha)\right)$ and

$$
\int_{E} \tau \pi^{*}(\alpha)=\int_{X} \pi_{*}\left(\tau \pi^{*}(\alpha)\right)=\int_{X} \pi_{*}(\tau) \alpha .
$$

We now identify $\pi_{*}(\tau)$. In equivariant cohomology this is straighforward since the equivariant Thom isomorphism gives

$$
e \pi_{*}(\beta)=i^{*}(\beta) \in h_{T}^{*}(X)=H^{*}(X)\left[u, u^{-1}\right]
$$

where $e$ is the equivariant Euler class. When we apply this to $\tau$ we see that

$$
\pi_{*}(\tau)=\frac{1}{e}
$$

indeed we may use this equation as a definition of the equivariant Euler class. The advantage of this point of view is that it will extend to give a definition of the equivariant Euler class of an infinite dimensional bundle provided we can define the operation $\pi_{*}$.

There is a slight imprecision here since the usual form of the Thom isomorphism involves forms of compact support. There are two simple ways around this. The first is to define forms of rapid decay on $E$ as in [14] and prove a Thom isomorphism using such forms. The second is to notice that $\pi_{*}(\tau)=\pi_{*}\left(\tau_{1}\right)$ where $\tau_{1}$ is defined above. But now $\tau_{1}$ has compact support and we may use the standard form of the Thom isomorphism.

The Thom class $U$ has the property that $\pi_{*}(U)=1$ in cohomology so it seems natural to use the name Thom/Euler class for the form $\tau$.

Suppose now that $Y$ is a finite dimensional compact closed $S^{1}$-manifold with an invariant metric and $\alpha \in \Omega_{T}^{*}(Y)\left[u, u^{-1}\right]$ is equivariantly closed. Let $E$ be the normal bundle to the fixed point set $F$ and $N=h(E)$ an invariant tubular neighbourhood of $F$. Then using Theorem 4.1 and the definition of $\sigma_{h}$

$$
\int_{Y} \alpha=\int_{Y} \sigma_{h}\left(i^{*}(\alpha)\right)=\int_{E} \tau \pi^{*}\left(i^{*} \alpha\right)
$$

The preceding discussion gives the exact integration formula of $[3,6,4,7]$ :

$$
\int_{Y} \alpha=\int_{F} \frac{i^{*}(\alpha)}{e} .
$$

where $e$ is the equivariant Euler class of $E$. 
Following [6, 4] this may be refined as follows. Since the circle acts on $Y$ by isometries the fixed point set $F$ is totally geodesic and the Riemannian connection on $Y$ induces an invariant connection on $E$, the normal bundle to the fixed point set. Let $R$ be the curvature of this connection on $E$. Let $L$ be the skew adjoint endomorphism of $E$ given by the infinitesimal action of the circle. Then in [6] and [4] it is shown that

$$
\pi_{*}(\tau)=\frac{1}{\operatorname{Pf}\left(\frac{R+u L}{2 \pi}\right)} \in \Omega^{*}(X)\left[u, u^{-1}\right]
$$

where Pf is the Pfaffian defined on matrices of differential forms. This gives the following integration formula for equivariantly closed forms $\alpha$ :

$$
\int_{Y} \alpha=\int_{F} \frac{i^{*}(\alpha)}{\operatorname{Pf}\left(\frac{R+u L}{2 \pi}\right)}
$$

We may interpret $(R+u L) / 2 \pi$ as the equivariant curvature of $E$ so that $\pi_{*}(\tau)$ is the Chern-Weil representative for $1 / e$ [13].

\section{LoOP SPACES AND THE $\hat{A}$-POLYNOMIAL}

Assume that $X$ is compact and finite dimensional and is equipped with a metric. We will explain the relation between the equivariant forms $\tau$ and the $\hat{A}$-polynomial of $X$. More precisley, we will show how to recover the ChernWeil representative for the $\hat{A}$-polynomial of $X$, defined by the metric, from the Thom/Euler class $\tau$ of the normal bundle of the space of constant loops inside the space of all loops. Our method is to combine the formula for $\pi_{*}(\tau)$ in finite dimensions with a calculation in [2].

The fixed point set of the circle action on $L X$ is the space $X$ considered as the space of constant loops; it has an invariant tubular neighbourhood (see the Appendix) and the normal bundle $N$ is the bundle over $X$ whose fibre at $x \in X$ is the space of loops in $T_{x} X$ whose first Fourier coefficient is zero. Let $N_{k}$ be the subbundle of $N$ whose fibre at $x$ consists of those loops in $T_{x} X$ whose (real) Fourier series

$$
\sum_{n \geq 0} a_{n} \sin n \theta+b_{n} \sin n \theta
$$

has the property that $a_{n}=b_{n}=0$ for $n \geq k$. Let $j_{k}: N_{k} \rightarrow N$ be the inclusion and $\pi_{k}: N_{k} \rightarrow X$ be the projection. Let

$$
\tau_{k}=j_{k}^{*}(\tau) \in \Omega^{*}\left(N_{k}\right)\left[u, u^{-1}\right]
$$

be the equivariant form associated to the bundle $N_{k}$ constructed in $\S 4$ and define

$$
\gamma_{k}=\left(\pi_{k}\right)_{*}\left(\tau_{k}\right) \in \Omega^{*}(X)\left[u, u^{-1}\right] .
$$

Since $\tau_{k}$ is homogeneous of degree 0 and integration along the fibres decreases degree by $2 m k$ it follows that $\gamma_{k}$ is homogeneous of degree $-2 m k$, where $m$ is the dimension of $X$. 
Let

$$
\hat{A}(u)=\sum_{i} a_{i} u^{-i} \in \Omega^{*}(X)\left[u, u^{-1}\right]
$$

be the element defined by taking $a_{i}$ to be the component in degree $2 i$ of the Chern-Weil representative for the $\hat{A}$-polynomial of $X$.

Theorem 5.1. Let $m$ be the dimension of $X$. The sequence $\left(k ! u^{k}\right)^{m} \gamma_{k}$ converges in the $C^{\infty}$ topology on $\Omega^{*}(X)\left[u, u^{-1}\right]$ and

$$
\lim _{k \rightarrow \infty}\left(k ! u^{k}\right)^{m} \gamma_{k}=\hat{A}(u)
$$

Proof. The $S^{1}$-bundle $N_{k}$ is a direct sum

$$
N_{k}=\bigoplus_{n=0}^{k} T_{c}(X)_{n}
$$

where $T_{c}(X)$ is the complexified tangent bundle of $X$ and the action of the circle on $T_{c}(X)_{n}$ is given by multiplication by $z^{n}$. Let $R$ be the curvature of $T_{c} X$ regarded as a two-form on $X$ with values in $\operatorname{End}\left(T_{c} X\right)$ and let $L$ be the skew adjoint endomorphism $L(v+i w)=w-i v$ of $T_{c} X$. Then (compare $\S 4$ )

$$
\gamma_{k}=\left(\pi_{k}\right)_{*}\left(\tau_{k}\right)=\prod_{n=1}^{k} \operatorname{Pf}\left(\frac{2 \pi n u L+R}{2 \pi}\right)^{-1}
$$

and since $T_{c} X$ has rank $m$ it follows that

$$
\left(k ! u^{k}\right)^{m}\left(\gamma_{k}\right)=\left(k ! u^{k}\right) \prod_{n=1}^{k} P f\left(\frac{2 \pi n u L+R}{2 \pi}\right)^{-1}=\prod_{n=1}^{k} P f\left(\frac{2 \pi n u L+R}{2 \pi u n}\right)^{-1} .
$$

The rest of the calculation follows Atiyah [2] and we summarise it very briefly. It is sufficient to assume that $T_{c} X$ splits into a sum of complex line bundles with curvatures $x_{i}$ and, since we are dealing with the complexification of a real bundle, the total Chern form is given by

$$
\prod_{i=1}^{d}\left(1+\frac{x_{i}^{2}}{4 \pi^{2}}\right)
$$

where $d$ is the greatest integer less than or equal to $m / 2$. Now we compute the Pfaffians occurring in (5.2) to get

$$
\gamma_{k}=P_{k}\left(x_{1}, \ldots, x_{d}\right)=\prod_{n=1}^{k} \prod_{i=1}^{d}\left(1+\frac{x_{i}^{2}}{(2 \pi n u)^{2}}\right) \text {. }
$$

Finally since

$$
\lim _{k \rightarrow \infty} P_{k}\left(x_{1}, \ldots, x_{d}\right)=\prod_{i=1}^{d} \frac{x_{i} / 2 u}{\sinh \left(x_{i} / 2 u\right)}
$$


uniformly on compact sets, as complex valued functions, it follows that

$$
\lim _{k \rightarrow \infty}\left(k ! u^{k}\right)^{m}\left(\pi_{k}\right)_{*}\left(\tau_{k}\right)=\frac{R / 2 u}{\sinh (R / 2 u)}
$$

in the $C^{\infty}$ topology on the space $\Omega^{*}(X)\left[u, u^{-1}\right]$.

Theorem 5.1 is one way to understand the statement that the renormalised equivariant Euler class of the bundle $N$ is $\hat{A}(u)^{-1}$. One thinks of the operation

$$
x \mapsto \lim _{k \rightarrow \infty}\left(k ! u^{k}\right)^{m}\left(\pi_{k}\right)_{*} j_{k}^{*}(x),
$$

when it is defined, as a renormalised integration along the fibres operation. It is defined when $x$ is the Thom/Euler class $\tau$ of the normal bundle to the space of constant loops inside the space of all loops and so, following the finite dimensional analogy, the equivariant Euler class of $N$ is $\hat{A}(u)^{-1}$.

\section{APPENDIX: THE GEOMETRY OF THE LOOP SPACE}

The smooth loop space is modelled on a Fréchet space. It is not true that any Fréchet manifold admits smooth partitions of unity or a metric. The loop space, however is modelled on the particular Fréchet space $C^{\infty}\left(S^{1}, V\right)$ where $V$ is a finite dimensional real vector space. The Fréchet topology is given by the family of seminorms

$$
\max _{t \in S^{1}}\left|f^{(k)}(t)\right|
$$

However, in view of the inequalities

$$
\left|f^{(k)}(t)\right|^{2}=\left|\int_{0}^{t} f^{(k+1)}(s) d s\right|^{2} \leq \int_{0}^{1}\left|f^{(k+1)}\right|^{2} d s
$$

we can use instead the family of $L^{2}$-norms

$$
\left\|f^{(k)}\right\|_{2}=\int_{0}^{1}\left|f^{(k)}(t)\right|^{2} d t
$$

Let $d$ be the metric defined by this family of $L^{2}$-norms. If $B$ is a smooth bump function on $\mathbb{R}$ centered at 0 , then $B(d(f,-))$ is a smooth bump function on $C^{\infty}\left(S^{1}, V\right)$ centered at $f$. Thus $C^{\infty}\left(S^{1}, V\right)$ has enough smooth bump functions to guarantee the existence of partitions of unity on $L X$, see $[1, \S 5.5]$.

A similar argument shows that any manifold with local model $C^{\infty}\left(S^{1}, V\right)$ admits a metric but for $L X$ it is easier to use a direct argument. Suppose $g(-,-)$ is a Riemannian metric on $X$. Let $\gamma$ be a loop in $X$ and let $W_{1}, W_{2}$ be vector fields along the loop $\gamma$, that is vectors in the tangent space $T_{\gamma} L X$. Then

$$
G\left(W_{1}, W_{2}\right)=\int_{0}^{1} g\left(W_{1}(t), W_{2}(t)\right) d t
$$

is the required positive definite inner product on the tangent space $T_{\gamma} L X$. We 
now construct an invariant tubular neighbourhood of the constant loops inside the space of all loops. Embed $X$ isometrically inside $\mathbb{R}^{n}$, then since $X$ is compact there exists $\varepsilon>0$ such that

(1) Any ball of radius $\varepsilon$ in $X$ is geodesically convex.

(2) The set $N_{\varepsilon}=\left\{x \in \mathbb{R}^{n} \mid d(x, X)<\varepsilon\right\}$ is an open tubular neighbourhood of $X$ in $\mathbb{R}^{n}$.

Now let $U$ be the open set in $L X$ such that any loop in $U$ has radius less than $\varepsilon$. Define a projection $\pi: U \rightarrow X$ as follows. Let $\sigma: L \mathbb{R}^{n} \rightarrow \mathbb{R}^{n}$ be the map which assigns to each loop its zero Fourier coefficient. Then if $\gamma$ is in $U$, $\sigma(\gamma)$ is in $N_{\varepsilon}$ and we define $\pi$ to be the composite of $\sigma$ with the projection $N_{\varepsilon} \rightarrow X$. Then $U$ is the required invariant tubular neighbourhood of $X$ in $L X$ and $\pi$ is the projection from the total space of this tubular neighbourhood onto $X$.

Another interesting example where the hypotheses of Theorem 2.1 can be verified by a similar argument is the space of based loops in a Lie group with its natural circle action.

\section{REFERENCES}

1. R. Abraham, J. E. Marsden and T. Ratiu, Manifolds, tensor analysis, and applications, Addison-Wesley, 1983.

2. M. F. Atiyah, Circular symmetry and stationary phase approximation, Colloque en l'honneur de Laurent Schwartz, Astérisque 131 (1985), 311 -323.

3. M. F. Atiyah and R. Bott, The moment map and equivariant cohomology, Topology 23 (1984), 1-28.

4. N. Berline and M. Vergne, Zéros d'un champ des vecteurs et classes charactéristiques équivariantes, Duke Math. J. 50 (1983), 539-548.

5. J.-M. Bismut, Index theorem and equivariant cohomology on the loop space, Comm. Math. Phys. 98 (1985), 213-237.

6. Localisation formulas, superconnections and the index theorem for families, Comm. Math. Phys. 103 (1986), 127-166.

7. J. J. Duistermatt and G. J. Heckmann, On the variation in the cohomology of the symplectic form on the reduced phase space, Invent. Math. 93 (1981), 139-149.

8. E. Getzler, J. D. S. Jones and S. B. Petrack, Cyclic homology, loop spaces and the Chern character, Operator Algebras and Applications, Vol. 1, edited by D. E. Evans and M. Takesaki, LMS Lecture Note Series 135, Cambridge Univ. Press, 1988, pp. 95-108.

9. E. Getzler, J. D. S. Jones and S. B. Petrack, Differential forms on loop spaces and the cyclic bar construction, Topology (to appear).

10. T. G. Goodwillie, Cyclic homology, derivations and the free loop space, Topology 24 (1985), 187-217.

11. J. D. S. Jones, Cyclic homology and equivariant homology, Invent. Math. 87, 403-423.

12. J. D. S. Jones and S. B. Petrack, Le théorème des points fixes en cohomologie équivariante en dimension infinie, C.R. Acad. Sci. Paris Ser. I 306 (1988), 75-78.

13. J. Milnor and J. Stasheff, Characteristic classes, Ann. of Math. Studies, no. 76, Princeton Univ. Press, Princeton, N. J., 1974.

14. V. Matthai and D. G. Quillen, Superconnections, equivariant differential forms and the Thom class, Topology 25 (1986), 85-110. 
15. C. Taubes, On the cohomology of elliptic genera, Preprint, Harvard University, 1987.

16. E. Witten, Supersymmetry and Morse theory, J. Differential Geom. 17 (1982), 661-692.

Mathematics Institute, The University of Warwick, Coventry CV4 7AL, England

UeR de Mathématiques de l'Université de Paris 7, 2 Place Jussieu, 75005 Paris, France 